\title{
Finkelstein's Test Is Superior to Eichhoff's Test in the Investigation of de Quervain's Disease
}

\author{
Feiran Wu ${ }^{1} \quad$ Asim Rajpura ${ }^{1} \quad$ Dilraj Sandher ${ }^{2}$ \\ ${ }^{1}$ Wrightington Hospital, Wigan, United Kingdom \\ 2Department of Orthopaedics and Trauma, Manchester Royal Infirmary, \\ Central Manchester University Hospitals, Manchester, United Kingdom \\ J Hand Microsurg 2018;10:116-118
}

\begin{abstract}
Address for correspondence Feiran Wu, MA, MBBChir, FRCS, Upper Limb Unit, Wrightington Hospital, Hall Lane, Appley Bridge, Wigan, WN6 9EP, United Kingdom (e-mail: feiran.wu@gmail.com).
\end{abstract}

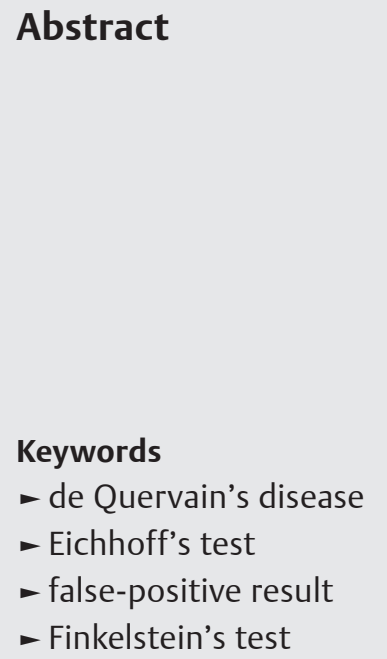

Introduction de Quervain's tenosynovitis is a common pathologic condition of the hand. Finkelstein's test has long been considered to be a pathognomonic sign of this diagnosis, yet most clinicians and instruction manuals erroneously describe what is in fact the Eichhoff's test, which is thought to produce similar pain by tendon stretching in a normal wrist. The purpose of this study was to compare Finkelstein's test with Eichhoff's test in asymptomatic individuals.

Materials and Methods Thirty-six asymptomatic participants (72 wrists) were examined using both Finkelstein's and Eichhoff's tests with a minimum interval of 24 hours between the tests.

Results The results showed that Finkelstein's test was more accurate than Eichhoff's test. It demonstrated higher specificity, produced significantly fewer numbers of false-positive results, and also caused significantly less discomfort to patients.

Conclusion This study recommends Finkelstein's test as the clinical examination of choice for the diagnosis of de Quervain's disease.

\section{Introduction}

de Quervain's tenosynovitis is a common pathologic condition affecting the first extensor compartment of the wrist, with an incidence of 0.94 per 1,000 person-years. ${ }^{1}$ The disease has been linked to people who perform manual work, owing to the unique mobility of the human thumb, ${ }^{2}$ with women four times as likely to be affected than men, and increased incidence in nonwhite individuals and those older than 40 years of age. ${ }^{1}$ Patients with this condition typically present with radial sided wrist pain and may have difficulty with lifting, grasping, and twisting activities involving the thumb. Other features in the diagnosis include signs of local swelling, tendon sheath thickening, and tenderness over the radial styloid.

Finkelstein's test has long been considered to be a pathognomonic sign of this diagnosis. ${ }^{3}$ However, most clinicians and instructional manuals describe what is in fact the Eichhoff's test. ${ }^{4-6}$ Errors in correctly describing Finkelstein's test can be traced to Leao (1958) who quoted Eichhoff's maneuver as Finkelstein's test. ${ }^{2}$ Eliott pointed out the mistake in 1992 and explained the difference between Finkelstein's test and its incorrectly described variant, which is thought to produce false-positive results by tendon stretching in normal individuals. ${ }^{5,7}$

Several other authors have also criticized the Eichhoff's test for producing positive results within normal individuals. ${ }^{5-7}$ However, to date, there are no published studies comparing the outcomes of the originally described Finkelstein's test with Eichhoff's variant. The intention of a clinical test is to guide investigation and management. If a clinical test has a high false-positive rate, it may lead to excessive investigation and treatment with the consequent costs and risks. This study aims to investigate whether Finkelstein's test is more accurate than Eichhoff's test.

\section{Materials and Methods}

A prospective controlled study was performed at the local university hospital. Over a 3-month period, asymptomatic individuals were invited to participate. Informed written consent was obtained from all participants. Patients were excluded if they suffered from a history of wrist pain, had received

October 8, 2017

accepted after revision

December 23, 2017

published online

March 20, 2018 
a previous diagnosis of de Quervain's tenosynovitis, had ever sustained a fracture of the distal radius, suffered from inflammatory arthropathy, or were diagnosed with intersection syndrome.

All patients underwent Finkelstein's and Eichhoff's tests as described in literature in both dominant and nondominant wrists. ${ }^{5}$ The tests were performed by the main author (F.W.) under supervision from A.R., both of whom worked in the upper limb unit. Each measurement was performed twice and the mean was recorded.

All participants underwent ultrasonography of both wrists prior to examination. No participants demonstrated radiographic evidence of tenosynovitis. There was a minimum interval of 24 hours between tests on the same wrist. Finkelstein's test was performed by placing the patient's wrist on the edge of a table. The examiner subsequently asked the patient to actively ulnarly deviate the wrist before grasping the patient's thumb and passively flexing it into the palm ( - Fig. 1). ${ }^{5}$ Eichhoff's test was performed by asking the participant to place the thumb within the hand and clench tightly with the other fingers. The hand was then passively abducted ulnarward by the examiner ( $\boldsymbol{- \text { Fig. }}$ 2). ${ }^{5}$ In both the tests, the examiner can also perform these maneuvers while

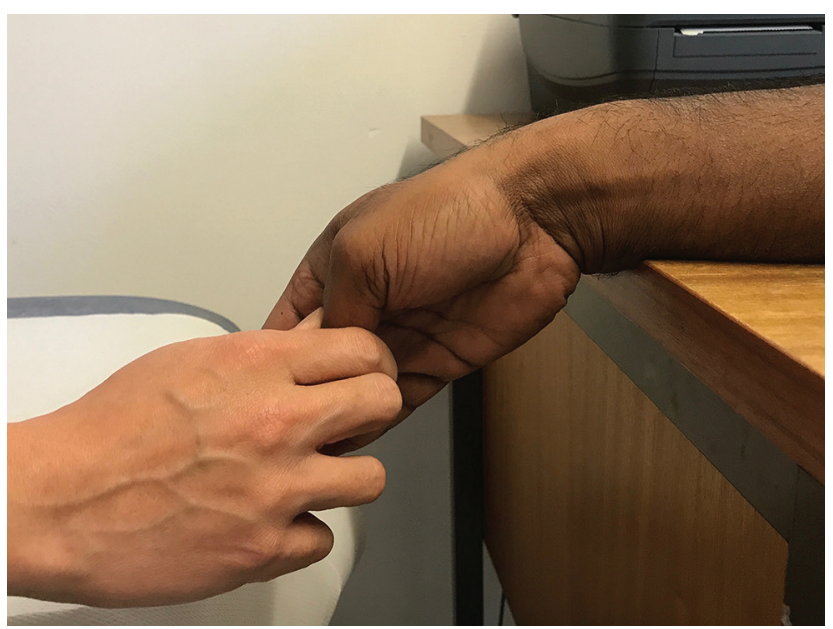

Fig. 1 Finkelstein's test on the patient.

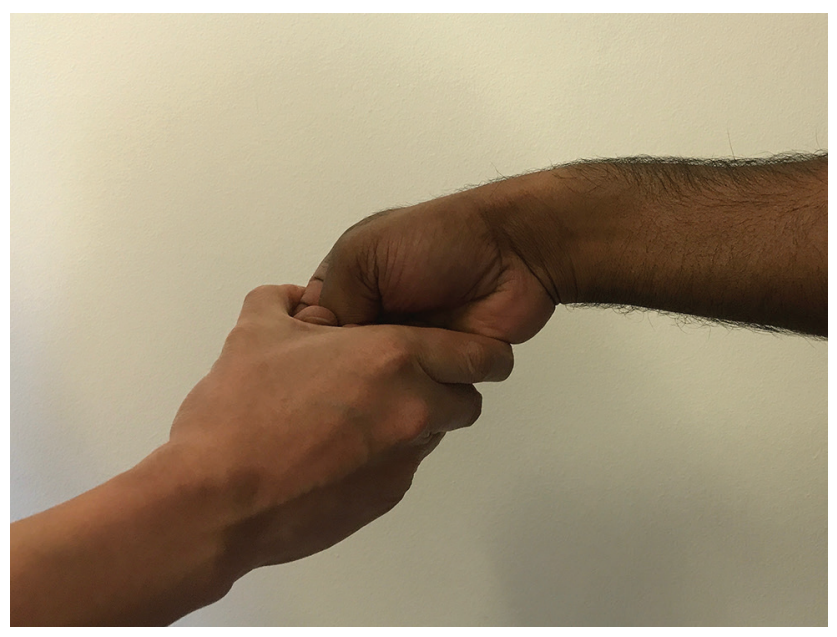

Fig. 2 Eichhoff's test on the patient. palpating the abductor pollicis longus and extensor pollicis brevis tendons over the lateral radius and feeling for moving nodularity, tendon rub, or popping directly over the tendon. Participants were asked to grade the degree of pain they experienced on a linear visual analogue scale (VAS) from 0 to 100 , with 0 being no pain and 100 being maximal pain. Findings of moderate or severe pain (44-100) was considered to be false positive. ${ }^{8}$

Results are given as the mean (standard deviation [SD]) or the median (range) as indicated by tests for normality. Comparisons between the two tests were made using the Wilcoxon signed-rank test. Statistical significance is defined as 0.05 .

\section{Results}

In total, 36 eligible patients ( 72 wrists) participated in the study. The mean age of the participants was 43 years (range: 24-66, SD: 12.7 years). Sixteen participants were males and 34 were right-hand dominant.

There were a total of eight false-positive results (five patients), all of which occurred from the Eichhoff's test. ( $p=0.003$, chi-square test). More false positives were encountered in the dominant hand (five dominant, three nondominant). Eichhoff's test also produced a significantly greater degree of discomfort than Finkelstein's test (Finkelstein's test: mean VAS 2.6, range: 0-41; Eichhoff's test: mean VAS 12.7, range: $0-87 ; p<0.01$ Wilcoxon signed rank test) (-Table 1).

The specificity of a test defines how good the test is at correctly excluding patients who do not have the condition under test. ${ }^{9}$ Finkelstein's test was more specific than Eichhoff's test, with a specificity of $100 \%$, compared with $89 \%$ for Eichhoff's test.

\section{Discussion}

This study confirmed that the hypothesis that Finkelstein's test is more accurate than Eichhoff's test and produced fewer false-positive results. Apart from being less specific, Eichhoff's test also produced a greater degree of discomfort than the Finkelstein test in patients.

The first description of the test to evaluate de Quervain's tenosynovitis was by Finkelstein in 1930, which stated that pain was elicited on traction of the thumb, which was worsened with ulnar deviation of the hand. ${ }^{3}$ Eichhoff's variant of this manoeuver involved the thumb gripped in the palm by the other fingers followed by passive ulnar deviation of the wrist with, which caused severe discomfort. ${ }^{4}$ Eichhoff used this maneuver to illustrate his understanding of the pathomechanics of the disease process, namely stretching the

Table 1 Test results

\begin{tabular}{|l|l|l|}
\hline & Finkelstein's test & Eichhoff's test \\
\hline False positives & 0 & 8 \\
\hline Mean VAS & 2.6 & 12.7 \\
\hline Range & $0-41$ & $0-87$ \\
\hline Specificity & $100 \%$ & $89 \%$ \\
\hline
\end{tabular}

Abbreviation: VAS, visual analogue scale. 
tendons and sheath of the first dorsal compartment as the cause for pain in de Quervain's tenosynovitis. This was to confirm his theory that repetitive tendon stretching by ulnar abduction of the hand could cause the condition. There is no evidence that Eichhoff meant this to be a test to diagnose de Quervain's tenosynovitis, yet Eichhoff's maneuver has been taught as Finkelstein's test in texts of hand surgery. ${ }^{10}$ The first time this is described incorrectly appears to the in the paper by Leao. ${ }^{2}$

Since the initial description of Finkelstein's test and Eichhoff's variant, there has been controversy regarding the accuracy of the tests and the generation of false-positive results in normal wrists. ${ }^{5-7}$ The mechanism for both the tests involves generation of a passive distension and shear stress between the tendons of the first dorsal compartment and the radius on its blunt styloid edge. Eichhoff's maneuver produces a greater degree of ulnar deviation of the wrist, because the patient's entire hand is abducted ulnarward by the examiner rather than just the thumb in Finkelstein's test. This would naturally create a greater level of distension and shear stress at the radial styloid, thereby causing pain in a normal wrist. Brunelli postulated that distension of the joints in Eichhoff's test can also create pain in other articular areas that is unrelated to true de Quervain's disease, owing to the tensioning of the radial collateral carpal ligament, the scaphotrapezial ligament, and the carpometacarpal ligament. ${ }^{7}$

This is the first study to demonstrate that in a normal population Finkelstein's test is more specific and produces significantly fewer false-positive results than Eichhoff's test. The authors recognize the limitations of the study. They do not have a group of patients with de Quervain's tenosynovitis to compare the sensitivity of the two tests. However, it is clear that Finkelstein's test produces fewer false-positive results and less patient discomfort, and therefore it should be the clinical test of choice when examining a patient suspected of having de Quervain's tenosynovitis, rather than the erroneous Eichhoff's variant. This will help reduce unnecessary further investigation or treatment.

\section{Funding}

None.

\section{Conflict of Interest}

None.

\section{References}

1 Wolf JM, Sturdivant RX, Owens BD. Incidence of de Quervain's tenosynovitis in a young, active population. J Hand Surg Am 2009;34(1):112-115

2 Leao L. De Quervain's disease; a clinical and anatomical study. J Bone Joint Surg Am 1958;40-A(5):1063-1070

3 Finkelstein $\mathrm{H}$. Stenosing tendovaginitis at the radial styloid process. J Bone Joint Surg 1930;509-540

4 Eichhoff E. Zur pathogenese der tendovaginitis stenosans. Bruns Beit Klin Chir. 1927;CXXXIX:746-755

5 Elliott BG. Finkelstein's test: a descriptive error that can produce a false positive. J Hand Surg [Br] 1992;17(4):481-482

6 Waseem M, Khan M, Hussain N, Giannoudis PV, Fischer J, Smith RM. Eponyms: errors in clinical practice and scientific writing. Acta Orthop Belg 2005;71(1):1-8

7 Brunelli G. [Finkelstein's versus Brunelli's test in de Quervain tenosynovitis] [in French] Chir Main 2003;22(1):43-45

8 Hawker GA, Mian S, Kendzerska T, French M. Measures of adult pain: Visual Analog Scale for Pain (VAS Pain), Numeric Rating Scale for Pain (NRS Pain), McGill Pain Questionnaire (MPQ), Short-Form McGill Pain Questionnaire (SF-MPQ), Chronic Pain Grade Scale (CPGS), Short Form-36 Bodily Pain Scale (SF-36 BPS), and Measure of Intermittent and Constant Osteoarthritis Pain (ICOAP). Arthritis Care Res (Hoboken) 2011;63(Suppl 11): S240-S252

9 Loong TW. Understanding sensitivity and specificity with the right side of the brain. BMJ 2003;327(7417):716-719

10 Lister G. The Hand. 3rd ed. New York, NY: Churchill Livingstone; 1993 